\title{
Sintomas de stress em estudantes moçambicanos do curso de Psicologia
}

\author{
Mário da Cruz Elias Matsinhe* \\ Antónia Maria Cândido* \\ Mussa Abacar** \\ Gildo Aliante***
}

\begin{abstract}
Resumo
Este estudo visou avaliar os sintomas de stress em estudantes do curso de Psicologia de uma Universidade pública da região sul de Moçambique, verificando possíveis associações com variáveis sociodemográficas e acadêmicas. O estudo envolveu uma amostra de 100 estudantes de graduação, sendo 78 do gênero feminino e 22 do gênero masculino, com a faixa etária entre 18 a 51 anos. Os dados foram coletados por meio de um questionário sociodemográfico e do Inventário de Sintomas de Stress de Lipp - ISSL. As análises estatísticas realizadas sugerem que 45\%dos estudantes investigados não apresentaram stress, 55\%revelaram sinais de stress, sendo 40\%na fase de resistência, 13\%na fase de exaustão e $2 \%$ na fase de alerta, com predomínio de sintomas de natureza psicológica. O perfil de estudantes com maior vulnerabilidade ao stress é constituído pelas seguintes características: ser do gênero feminino, ter idade entre 18 a 25 anos, ser solteiro, estar no $3^{\circ}$ no do curso, e não possuir vínculo empregatício. Em conclusão, os resultados permitem considerar a presença de um quadro instalado de stress nos estudantes investigados, ressaltando a importância das variáveis sociodemográficas e acadêmicas na percepção do fenômeno. Assim, torna-se necessária a implantação de serviços de assistência e de atenção psicossocial aos estudantes na Universidade.
\end{abstract}

Palavras-chave: Stress; Saúde mental; Ensino superior

\section{Stress symptoms of Mozambican psychology students}

\begin{abstract}
This study aimed to evaluate the symptoms of stress in students of the Psychology course at a public University in the southern region of Mozambique, verifying possible associations with sociodemographic and academic variables. The study involved a sample of 100 undergraduate students, 78 of which were female and 22 were male, aged between 18 and 51 years. Data were collected using a sociodemographic questionnaire and the Lipp Stress Symptoms Inventory - LSSI. The statistical analyzes carried out suggest that $45 \%$ of the investigated students did not present stress, $55 \%$ showed signs of stress, $40 \%$ in the resistance phase, $13 \%$ in the exhaustion phase and $2 \%$ in the alert phase, with a predominance of psychological symptoms. The profile of students with greater vulnerability to stress consists of the following characteristics: being female, being between 18 and 25 years old, being single, being in the $3 \mathrm{rd}$ in the course, and not having a job. In conclusion, the results allow considering the presence of an installed picture of stress in the investigated students, emphasizing the importance of sociodemographic and academic variables in the perception of the phenomenon. Hence, it is necessary to implement psychosocial assistance and care services for students at the University.
\end{abstract}

Keywords: Stress; Mental health; University education.

* Graduação em andamento em Psicologia Social e das Organizações - Universidade Pedagógica de Maputo - Moçambique

** Doutor em Psicologia Cognitiva pela UFP, Mestre em Psicologia das Organizações, Social e do Trabalho Universidade do Porto, Psicólogo pela Universidade Pedagógica de Maputo - Moçambique. Prof. de Psicologia, Faculdade de Educação e Psicologia na Universidade Rovuma - Moçambique

*** Doutorando e Mestre em Psicologia Social e Institucional - UFGRS. Graduado em Planificação pela Universidade Pedagógica-Moçambique. Bolsista Capes. 


\section{Introdução}

$\mathrm{O}$ ingresso na Universidade constitui para muitos cidadãos moçambicanos um enorme desafio, devido à limitada expansão da rede das Instituições do Ensino Superior (IES) ou a falta de condições financeiras para os candidatos custearem as despesas relativas aos estudos, estadia e alimentação. Até o presente, Moçambique conta com 53 instituições de ensino superior, entre Universidades, Institutos Superiores, Escolas Superiores e Academias, sendo 22 públicas e 31 privadas. Maior parte dessas instituições está localizada nas principais cidades moçambicanas nomeadamente, Maputo, Beira e Nampula, situadas nas regiões sul, centro e norte respectivamente.

Entre as IES públicas e privadas, as do ensino público têm sido as de maior procura devido às suas características, pois são consideradas mais acessíveis do ponto de vista financeiro. Ser aprovado em uma instituição do ensino superior público constitui um importante acontecimento na vida do candidato e da sua família. Apesar disso, ao longo de vida acadêmica alguns estudantes podem enfrentar problemas e dificuldades de adaptação ao novo ambiente escolar em virtude de demandas relacionadas ao curso e à instituição e de lidar com as várias obrigações e exigências típicas do ambiente acadêmico, às relações interpessoais, à quebra de expectativas, ao excesso de carga horária, à falta de domínio de línguas, de algumas disciplinas e conteúdos, aos problemas financeiros, ao isolamento familiar, às reprovações (Carleto, Moura, Santos, \& Pedrosa, 2018). Todos esses aspectos podem se tornar em stressores acadêmicos e, por conseguinte, originar problemas de saúde mental e física.

Vale mencionar que a produção, disseminação e assimilação do conhecimento mobilizam aspectos cognitivos, sociais, físicos e emocionais que percorrem todo o processo da formação acadêmica (Moretti \& Hubner, 2017). Se, de acordo com Siqueira et al. (2017), o contexto universitário proporciona um ambiente de desafios, instigados pela quebra de paradigmas de aquisição de conhecimentos e estabelecimento de relações interpessoais, seja pela pluralidade e volume de conteúdos a serem estudados, complexidade das disciplinas e temas, bem como pela insegurança referente ao futuro, podendo favorecer a ocorrência do stress nos estudantes.

Em Moçambique, um estudo com amostras de estudantes de uma universidade pública revelou que a sobrecarga de matéria ou de trabalhos acadêmicos, as dificuldades financeiras e econômicas, as dificuldades na assimilação de conteúdo, o precário relacionamento social, as situações familiares, a atuação dos docentes, o reduzido sucesso acadêmico, o número de disciplinas reprovadas e de vezes que o aluno reprovou, as condições físicas, a fraca preparação acadêmica dos professores, a falta de domínio da língua inglesa e as práticas de avaliação foram aspectos críticos de satisfação acadêmica e principais stressores (Salomão, Abacar, \& Aliante, 2018). Diversos estudos (Aliante, Abacar, Saquina, \& Aristides, 2019; ANDIFES, 2019; Ariño \& Bardagi, 2018; Assis, Silva, Lopes, Silva, \& Santani, 2013; Cestari, Barbosa, Florêncio, Pessoa, \& Moreira, 2017; Estrela, Rezende, Guedes, Pereira, \& Souza, 2018; Lima, Soares, Prado \& Albuquerque, 2016; Medeiros, Camargo, Barbosa, \& Calderia, 2018; Querido, Naghettini, Orsini, Bartholomeu, \& Montie, 2016; Vieira \& Schermann, 2015) revelaram que os stressores acadêmicos podem originar vários problemas de saúde mental como ansiedade, depressão, stress e burnout, o que pode comprometer a qualidade de vida e sucesso acadêmico do estudante. Sendo assim, a pesquisa sobre o stress em estudantes assume uma importância central no contexto acadêmico.

Existem várias definições conceituais de stress. O stress é, segundo Burrows (2004), o resultado da discrepância entre uma exigência (biológica ou psicológica) apresentada e a capacidade do indivíduo para lidar com a mesma. Silva e Salles (2016) concebem o stress como uma resposta aos vários estímulos aos quais os sujeitos estão submetidos e às cobranças a elas impostas, podendo manifestar-se de um conjunto de reações psicológicas ou fisiológicas que alteram o organismo, tentando se adaptar as mudanças que surgem no dia a dia. E Moretti e Hubner (2017), entendem que o stress constitui um conjunto de reações do organismo quando deparado com uma situação de esforço físico, emocional, psicológico e que causa certo desconforto social, emocional, psicológico e físico.

As reações ao stress são naturais e até necessárias para a própria vida, e este assume grande importância no cotidiano do ser humano, influenciando a pessoa em todas as esferas do seu funcionamento (Nogueira \& Gomes, 2014). Todavia, sob algumas circunstâncias, essas reações podem tornar-se prejudiciais ao funcionamento do organismo (Limongi-França \& Rodrigues, 2009). Neste contexto, Selye (1993) propôs dois tipos de stress: o eustress e distress, em que o eustress constitui stress que suscita uma resposta adaptativa pelo organismo (stress positivo) e o distress (stress negativo) refere-se ao estímulo forte suscetível de provocar prejuízos no organismo (Nodari, Flor, Ribeiro, \& Carvalho, 2014).

No eustress, o esforço de adaptação gera sensação de realização pessoal, bem-estar e satisfação das neces- 
sidades, mesmo que decorrentes de esforços inesperados. É um esforço sadio na garantia de sobrevivência (Limongi-França \& Rodrigues, 2009). O eustress (euphoria + stress) significa o stress saudável, agradável e curativo. Assim, o stress em doses moderadas (eustress) fornece motivação e o aumento da produtividade; mas em doses excessivas (distress) resulta em destruição e desequilíbrio orgânico, prejudicando a qualidade de vida, atingindo a área social, da saúde e profissional (Lipp, 2004). A palavra distress contém o prefixo latim dis, que significa "mau ou ruim" e refere-se à tensão com rompimento do equilíbrio biopsicossocial por excesso ou falta de esforço, incompatível com tempo, resultados e realização. Desse modo, o distress traduz-se em resultados patológicos, negativos, destrutivos de eventos stressantes (Abacar, 2011). No contexto do presente artigo, o termo stress é usado como sinônimo do stress negativo (distress).

Para Limongi-França e Rodrigues (2009), a Síndrome de Adaptação Geral (General Adaptation Syndrome - GAS), proposta por Hans Selye, se desenvolve em três fases diferentes, nomeadamente: de alerta, resistência e exaustão. No entanto, em Lipp (2016), uma pesquisadora brasileira de reconhecido mérito nos estudos sobre o stress, é descrita uma quarta fase designada de quase-exaustão. Esta se situa entre a fase de resistência e de exaustão.

A fase de alarme ocorre após a exposição ao fator de stress, os processos homeostáticos são interrompidos e ocorrem rápidas alterações fisiológicas e neuroquímicas (tensão arterial, ritmo cardíaco, níveis de glicose e equilíbrio eletrolítico) que desencadeiam as "respostas de choque" ou "contra-choque", através do aumento da libertação de corticoesteróides pelo córtex suprarrenal e de adrenalina pela medula suprarrenal, como tentativa de contrariar estas alterações sentidas (Almeida, Brito-costa, Alberty, Gomes, Lima \& Castro, 2016). Há um aumento da produtividade do indivíduo, na medida em que aumenta a motivação para enfrentar uma determinada situação. Nessa fase, são frequentes os seguintes sinais de stress: irritabilidade, ansiedade, insônia, esquecimento e impossibilidade para se concentrar (Lipp, 2016). Se o fator de stress persistir, desenvolve-se uma segunda fase, a de resistência.

$\mathrm{Na}$ fase da resistência o organismo atinge uma adaptação à ameaça do meio e aos efeitos nocivos do stressor, o que mantém a contínua ativação fisiológica e neuroquímica embora em menor grau, comparativamente com o estado inicial anterior - e o aumento do funcionamento do eixo hipófise-suprarrenal, tornando o organismo mais vulnerável aos efeitos nefastos de outros fatores de stress (Almeida et at., 2016). Se o indivíduo não consegue restabelecer o equilíbrio, surge o stress. É uma fase caracterizada por fadiga, isolamento social, letargia, e até consumo de bebidas alcoólicas e café. Nesta fase o indivíduo utiliza toda a energia que tem à disposição para recuperar o equilíbrio perdido; caso este consiga restabelecer o equilíbrio, ele consegue superar a situação de stress antes que seja tarde demais, e de contrário fica propenso em desenvolver a fase posterior, a de quase-exaustão.

A fase de quase-exaustão é caracterizada pelo enfraquecimento do organismo e início do processo da doença. Precisa-se, então, de ajuda tanto médica para tratar o problema físico que já surgiu, como psicológica para aprender a lidar com a causa do stress. Devido ao enfraquecimento do organismo, doenças que permaneciam latentes são desencadeadas. Caso o stressor permaneça presente e o indivíduo não consiga lidar com ele adequadamente, ocorrerá a fase de exaustão (Lipp, 2016).

A fase de exaustão ou esgotamento é o estado patológico (Abacar, 2011). O indivíduo esgotou todas as formas que conhecia para lutar contra o agente causador de stress e cai em uma situação de completo desgaste, uma situação de exaustão física e mental que podem levar o indivíduo ao colapso. Dentre as consequências deste estado, aponta-se a doença física, a incapacidade de se concentrar, a maior irritabilidade, a perda de contato com a realidade ou ainda a morte (Lipp, 2016). Outros sinais relatados são a tristeza crônica, depressão, fadiga física e mental crônica, problemas estomacais, isolamento e pensamentos autodestrutivos.

A prevalência do stress no mundo é alta e extremamente preocupante (Lipp, Costa, \& Nunes, 2017), pois este constitui um dos mais importantes problemas de saúde mental que afeta pessoas de diversas regiões do mundo. Infelizmente, o stress em estudantes é um tema pouco debatido e investigado em Moçambique, tendo sido identificado apenas um estudo de Aliante, Abacar, Saquina \& Aristides (2019). Ademais, Martins, Silva, Maroco e Campos (2019) observaram que a maioria dos estudos que buscam investigar stress em universitários está voltada para os estudantes de medicina, sendo escassos os trabalhos que envolvem estudantes das demais áreas da Saúde e das Ciências Humanas e Exatas. Estes autores alertaram ainda que apesar de haver uma cobrança diferenciada em relação ao curso de Medicina, não se devem desconsiderar os estudantes de outros cursos. $\mathrm{Na}$ verdade, o interesse em pesquisar o construto stress em estudantes deriva das vivências, experiências dos dois primeiros autores que durante a sua formação em Psicologia na Universidade 
estudada, constataram constantes situações de reclamações, insegurança, ansiedade, medo e outros sentimentos negativos por parte dos seus colegas devido às diversas exigências acadêmicas, as quais devem ser investigadas.

Nesse contexto, o objetivo do estudo foi avaliar os sintomas de stress em estudantes do curso de Psicologia de uma Universidade pública da região sul de Moçambique, verificando possíveis associações com variáveis sociodemográficas e acadêmicas. A partir desse objetivo foram definidas as seguintes questões de estudo: a) qual é o nível de stress em estudantes do curso de Licenciatura em Psicologia de uma Universidade pública? b) Quais são os sintomas mais regulares nos estudantes do ensino superior? e, c) Como as variáveis sociodemográficas e acadêmicas interferem na ocorrência do stress entre os estudantes?

\section{Material e método}

Para o desenvolvimento deste estudo utilizou-se uma pesquisa quantitativa, de natureza descritiva. O estudo envolveu uma amostra não probabilística por acessibilidade de 100 estudantes que cursam Psicologia em Universidade pública localizada na região sul de Moçambique. A coleta de dados foi realizada na sala de aula no mês de outubro de 2019, de forma individual e os participantes foram informados sobre as pretensões da pesquisa, respectivos benefícios e riscos em participar da mesma.

Vale salientar que a Universidade onde foi realizada a pesquisa não possui um Comitê de Ética de Pesquisa Institucional. E para efeitos de realização desta pesquisa, os autores apresentaram uma Carta credencial emitida pela Direção da Faculdade de Educação e Psicologia da Universidade Pedagógica - Maputo, a qual apresentava o estudo e solicitava a sua realização. Obtida a autorização, os estudantes que consentiram em participar da pesquisa receberam as instruções dadas pelos dois primeiros autores, que coletaram os dados. Foram respeitados todos os procedimentos éticos em relação à pesquisas com seres humanos, nomeadamente o consentimento livre e esclarecido, o anonimato dos participantes, a confidencialidade dos dados e a proteção dos sujeitos de possíveis danos psicológicos. Caso algum participante se sentisse desconfortável durante a realização da pesquisa, teria o devido acompanhamento do psicólogo responsável pela pesquisa.

Os dados foram coletados por meio de um questionário de dados sociodemográficos e acadêmicos e, o Inventário de Sintomas de Stress para Adultos de Lipp (ISSL). O questionário de dados sociodemográficos tinha por objetivo coletar informações pessoais (idade, gênero e estado civil), e acadêmicas (regime, tipo de vínculo empregatício e ano de frequência do curso).

O Inventário de Sintomas de Stress para Adultos de Lipp (ISSL) é um instrumento de avaliação de stress, da autoria de Lipp (2000). Trata-se de um questionário que avalia a sintomatologia do stress, apresentando quadros com sintomas tanto de stress físico quanto psicológico, que o sujeito assinala se ocorreram durante determinados períodos de tempo, bem como em que fase de stress este se encontra, de acordo com o modelo quadrifásico do stress proposto pela autora (alerta, resistência, quase-exaustão e exaustão). Também o instrumento permite observar o tipo de sintoma predominante, físico ou psicológico.

O ISSL é constituído por 53 itens, dos quais 34 são referentes aos sintomas físicos e 19 aos psicológicos, dividindo-se em três partes. A primeira parte indica sintomas sentidos nas últimas 24 horas e é composta por 15 sintomas, sendo 12 sintomas físicos e três psicológicos. $\mathrm{Na}$ segunda parte são apontados 15 sintomas experimentados na última semana, dos quais 10 físicos e cinco psicológicos. Finalmente, a terceira e última parte abrange 23 sintomas sentidos no último mês, composto por 12 físicos e 11 psicológicos. A avaliação das respostas dadas pelos indivíduos é feita por meio do uso de tabelas do próprio manual do ISSL que transformam os dados brutos em porcentagem (Lipp, Costa \& Nunes, 2017; Souza, Santana, Pedra, Dias, Henrique \& Dantas, 2015).

O respondente é solicitado a indicar se tem apresentado o sintoma de stress especificado em cada quadro em 24 horas (Q1-parte um), uma semana (Q2-parte dois) ou um mês (Q3-parte três). A presença de stress pode ser constatada se qualquer um dos escores brutos atingir os limites determinados (maior que seis no Q1- parte um, ou seja, a soma de quantidade de sintomas de F1 e P1; maior que três no Q2- parte dois, soma de F2 e P2; e maior que oito no Q3-parte três soma de F3 e P3). Caso os escores obtidos estejam acima dos limites nos três quadros, é identificado o processo de agravamento do stress. Para definir a fase de stress em que o indivíduo se encontra, é considerado o quadro em que o mesmo mais pontuou, em termos porcentuais, sendo que segundo Faiad, Matsunaga, Rodrigues \& Rosa (2018), esses resultados permitem saber em que fase do stress a pessoa se encontra e se os sintomas que prevalecem são mais físicos ou psicológicos.

O uso do ISSL como medida para avaliar stress em estudantes neste estudo foi justifica-se por estar redigido em língua portuguesa e apresentar valor de alfa de 
Cronbach de 0,91 para a escala geral e, portanto, os itens refletirem um alto valor para o conceito intencional, qual seja: medir o nível de stress (Chaves, Souza, Silva, Oliveira, Lipp \& Pinto, 2016; Lipp, Costa \& Nunes, 2017). Além de o ISSL revelar uma boa consistência interna, a escolha desse instrumento se deveu, também, ao fato de ele ser uma ferramenta mais utilizada na medição dos sintomas de stress (Souza et al., 2015), principalmente nos países de língua portuguesa.

A análise dos dados foi realizada com uso de software estatístico SPSS (Statistical Package for Social Sciences), versão 20. Para determinar o perfil dos participantes e identificar os sintomas de stress, foi utilizada a estatística descritiva, observando as frequências das respostas nos itens da escala. Foram calculadas as frequências (absolutas e relativas) para determinar os sintomas mais regulares e as respectivas fases prevalecentes que o instrumento compõe.

\section{Resultados}

\section{Caracterização da amostra}

A amostra deste estudo foi constituída por 100 alunos, do curso de Psicologia da Universidade pesquisada, dos quais 78 eram do gênero feminino e 22 do gênero masculino, com idades compreendidas entre 18 a 51 anos. Quanto ao estado civil, 87 eram solteiros e 13 casados. Em relação à variável vínculo laboral, 78 não trabalhavam e 22 estavam empregados. No que diz respeito ao ano de frequência, 27 estavam no $1^{\circ}$ ano, 14 no $2^{\circ}, 36$ no $3^{\circ}$ e 23 no $4^{\circ}$ ano.

Frequência dos sintomas de stress

Para verificar os sintomas mais regulares na fase de alerta, resistência, quase-exaustão e exaustão foi feito o cálculo das frequências (absolutas e relativas) dos sintomas expostos no ISSL.

Tabela 1: Sintomas de stress nos estudantes

\begin{tabular}{|c|c|}
\hline Sintomas físicos & $\mathrm{n}$ \\
\hline Mãos e pés frios & 10 \\
\hline Boca seca & 28 \\
\hline Nó no estômago & 23 \\
\hline Aumento da sudorese & 6 \\
\hline Tensão Muscular & 23 \\
\hline Aperto da mandíbula/Ranger dos dentes & 5 \\
\hline Diarréia passageira & 15 \\
\hline Insônia & 28 \\
\hline Taquicardia & 6 \\
\hline Hiperventilação & 2 \\
\hline Hipertensão arterial súbita e passageira & 15 \\
\hline Mudança de apetite & 49 \\
\hline Sintomas psicológicos & $\mathrm{n}$ \\
\hline Aumento súbito de motivação & 32 \\
\hline Entusiasmo súbito & 16 \\
\hline Vontade súbita de iniciar novos projetos & 48 \\
\hline Sintomas físicos & $\mathrm{n}$ \\
\hline Problemas com a memória & 28 \\
\hline Mal-estar generalizado, sem causa específica & 27 \\
\hline Formigamento nas extremidades & 11 \\
\hline Sensação de desgaste físico constante & 47 \\
\hline Mudança de apetite & 44 \\
\hline Aparecimento de problemas dermatológicos & 12 \\
\hline Hipertensão arterial & 13 \\
\hline Cansaço constante & 60 \\
\hline Gastrite, úlcera ou indisposição estomacal muito prolongada & 22 \\
\hline Tontura ou sensação de estar flutuando & 28 \\
\hline Sintomas psicológicos & $\mathrm{n}$ \\
\hline Sensibilidade emotiva excessiva & 11 \\
\hline
\end{tabular}

\begin{tabular}{|c|c|}
\hline Dúvida quanto a si próprio & 39 \\
\hline Pensar constantemente em um só assunto & 53 \\
\hline Irritação excessiva & 38 \\
\hline Diminuição da libido & 11 \\
\hline Sintomas fisicos & $\mathrm{n}$ \\
\hline Diarréia frequente & 9 \\
\hline Dificuldades sexuais & 15 \\
\hline Insônia & 29 \\
\hline Náuseas & 14 \\
\hline Tiques & 11 \\
\hline Hipertensão arterial continuada & 13 \\
\hline Problemas dermatológicos prolongados & 6 \\
\hline Mudança estrema de apetite & 30 \\
\hline Excesso de gases & 23 \\
\hline Tontura frequente & 25 \\
\hline Úlcera, colite ou outro problema digestivo sério & 7 \\
\hline Enfarto & 3 \\
\hline Sintomas psicológicos & $\mathrm{n}$ \\
\hline Impossibilidade de trabalhar & 6 \\
\hline Pesadelos frequentes & 13 \\
\hline Sensação de incompetência em todas as áreas & 12 \\
\hline Vontade de fugir de tudo & 38 \\
\hline Apatia, depressão ou raiva prolongada & 23 \\
\hline Cansaço mental constante excessivo & 44 \\
\hline Pensar e falar constantemente em um só assunto & 31 \\
\hline Irritabilidade frequente sem causa aparente & 20 \\
\hline Angústia ansiedade, medo diariamente & 33 \\
\hline Hipersensibilidade emotiva & 9 \\
\hline Perda de senso de humor & 29 \\
\hline
\end{tabular}

Fonte: Resultados da pesquisa. 
Com base na Tabela 1, os sintomas físicos mais pontuados na fase de alerta foram: mudança de apetite; boca seca e insônia. $\mathrm{Na}$ fase de resistência foram evidenciados o cansaço constante, sensação de desgaste físico constante, mudança de apetite, problemas com a memória, tontura ou sensação de estar flutuando e mal-estar generalizado sem causa específica. Por fim, na fase de exaustão destacam-se mudança extrema de apetite, insônia e tontura frequente. Em relação aos sintomas psicológicos, os mais apontados foram sucessivamente vontade súbita de iniciar novos projetos e aumento súbito de motivação (fase de alerta), pensar constantemente em um só assunto, dúvida quanto a si próprio e irritação excessiva (fase de resistência) e cansaço constante excessivo, vontade de fugir de tudo, angústia ansiedade, medo diariamente, pensar e falar constantemente em um só assunto e perda de senso de humor (fase de quase-exaustão e exaustão).

Ocorrência de sintomas de stress em relação às variáveis sociodemográficas e acadêmicas

Com vista a avaliar a ocorrência de stress em função das variáveis sociodemográficas e acadêmicas (gênero, idade, ano de frequência, vínculo empregatício e estado civil) foram achadas frequências absolutas e relativas. $\mathrm{Na}$ Tabela 2 são apresentados os resultados alcançados.

Observa-se, na Tabela 2, que em termos absolutos as estudantes revelaram uma tendência de sofrer stress na fase de resistência, mas em termos relativos os homens obtiveram maior frequência de sintomas dessa fase. $\mathrm{O}$ cenário se verifica na variável ano de frequência, que em termos absolutos os estudantes do $1^{\circ}$ e $3^{\circ}$ sinalizaram muitos sintomas, mas em termos relativos, os alunos do $2^{\circ}$ e $4^{\circ}$ é que se mostraram muito vulneráveis ao stress na fase de resistência.
$\mathrm{Na}$ variável de estado civil, os solteiros tiveram maior frequência de sintomas presentes na fase de resistência que os casados. E no que tange o vínculo empregatício, os estudantes sem vinculação laboral são os que apresentam maior número de sintomas do que os que trabalham. E finalmente, na variável idade, os estudantes mais jovens (18 a 25 anos de idade) revelaram maior probabilidade de sofrer de stress quando comparados com os mais velhos (26 a 51 anos de idade).

\section{Fases do stress}

No prosseguimento das análises procurou-se identificar as fases do stress nos estudantes.

No Gráfico 1 é possível verificar os percentis obtidos nas diferentes fases de stress. Observa-se que 45\% dos participantes não demonstram sofrer de stress e 55\% sofrem deste em seus diferentes estágios: alerta, resistência e exaustão.

Gráfico 1: Fases do stress em estudantes

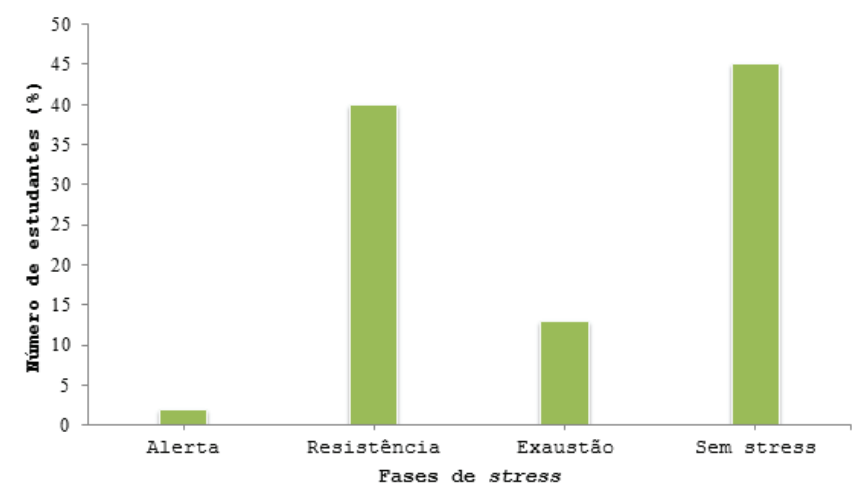

Fonte: Resultados da pesquisa.

Tabela 2: Ocorrência do stress em função das variáveis gênero, ano de frequência, estado civil, vínculo empregatício e idade

\begin{tabular}{|c|c|c|c|c|c|}
\hline \multirow[t]{2}{*}{ Fases do stress } & Gênero & Ano de Frequência & Estado Civil & $\begin{array}{c}\text { Vínculo } \\
\text { Empregatício }\end{array}$ & \multirow{2}{*}{$\begin{array}{l}\text { Idade } \\
18 \text { a } 25 \\
\end{array}$} \\
\hline & Masc. Fem. & $1^{\circ} \quad 2^{\circ} \quad 3^{\circ} \quad 4^{\circ}$ & Solt. Cas. & Com Sem & \\
\hline Sem stress & $08 \quad 12$ & $\begin{array}{llll}04 & 17 & 12 & 37\end{array}$ & $08 \quad 13$ & 32 & 27 \\
\hline Alerta & $02 \quad 00$ & $\begin{array}{llll}01 & 01 & 00 & 03 \\
\end{array}$ & 00 & 01 & 01 \\
\hline Resistência & $11 \quad 09$ & $\begin{array}{llll}09 & 14 & 08 & 35 \\
\end{array}$ & 04 & 27 & 16 \\
\hline Exaustão & 06 & $\begin{array}{llll}00 & 04 & 03 & 12\end{array}$ & 01 & 11 & 03 \\
\hline Total & 27 & $\begin{array}{llll}14 & 36 & 23 & 87\end{array}$ & 13 & 72 & 47 \\
\hline
\end{tabular}

Fonte: Resultados da pesquisa 


\section{Discussão}

Nesta investigação verificou-se a ocorrência de sintomas de stress de natureza física e psicológica. Destaca-se que maior parte dos participantes (55\%) demonstrou estar a sofrer de stress, sucessivamente nas fases de resistência (40\%), exaustão (13\%) e alerta (2\%). O estudo de Chaves et al. (2016) apontou que dos 207 estudantes envolvidos, $43 \%(n=89)$ não apresentaram stress e $57 \%(n=118)$ apresentaram sintomatologia da doença. Somando-se aos resultados do mesmo estudo, dos 57\%, 18\% ( $\mathrm{n}=21)$ estudantes se encontravam na fase de alerta, $60 \%(\mathrm{n}=71)$ na fase de resistência, $6,7 \%(\mathrm{n}=8)$ na fase de quase exaustão e $15,3 \%(n=18)$ na fase de exaustão, sendo a fase de resistência a mais predominante. No mesmo sentido, Querido et al. (2016) identificaram dos 50 estudantes, $52 \%$ apresentava stress com predominância de sintomas psicológicos e numa fase potencialmente adoecedora, mas pouco vista como tal (fase de resistência). Esses estudos se assemelham com os resultados da presente pesquisa, em que $55 \%$ apresentaram stress.

$\mathrm{Na}$ pesquisa de Moretti e Hübner (2017), 45,5\% do total da amostra $(n=101)$ revelou sintomas da fase de resistência. E na investigação de Costa et al. (2018) a prevalência de stress entre 173 estudantes de graduação em enfermagem foi de 73,3\%, com 65,7\% na fase de resistência do stress. Essa observação parece revelar que o curso de enfermagem propicia maior stress entre os estudantes.

No que diz respeito aos sintomas de stress, registrou-se a ocorrência de ambos os sintomas, quer de natureza física quer de ordem psicológica, em todas as fases da doença. No entanto, o fato de ter-se registrado sintomas de natureza psicológica tanto na fase de resistência como na exaustão, constitui um indicativo de maior risco de agravamento do stress. Estes resultados são similares aos do estudo de Aguiar et al. (2009) que apontou a predominância de sintomas psicológicos do stress em detrimento dos sintomas físicos. Igualmente, em relação à sintomatologia, Costa et al. (2018) verificaram o predomínio de sintomas psicológicos (42,0\%).

Quanto à influência das variáveis sociodemográficas e acadêmicas na percepção do stress, os resultados indicam que os estudantes do gênero feminino, do $3^{\circ}$ ano, solteiros, sem vínculo empregatício e com idades entre 18 a 25 anos são os que se mostram mais propensos ao stress. Estes resultados poderiam estar relacionados ao fato dos estudantes mais velhos já possuírem mais estrutura emocional, advinda de maior maturidade inclusive, em termos de experiência de vida e de lidar com situações stressoras, do que os estudantes mais novos, o que pode ser um fator protetor. Importa ressaltar ainda que a maioria dos estudantes mais velhos que fazem parte da amostra é simultaneamente da classe trabalhadora, carregando consigo esperanças em termos de crescimento e desenvolvimento profissional por meio da mudança de carreira e, por conseguinte, a melhoria das condições de vida.

Cabe ainda apontar que, tal como constataram Aliante et al. (2019), a falta de vínculo empregatício se associou ao stress nos estudantes pesquisados. Estes achados corroboram parcialmente com os de outros estudos anteriores. A título ilustrativo, Bayram e Bilgel (2008), revelaram escores médios de stress em estudantes na faixa etária entre 20 e 26 anos. No estudo de Bublitz, Guido, Lopes e Freitas (2016), verificou-se que a faixa etária associou-se com os altos níveis de stress, sendo que os estudantes da faixa etária entre 20 e 24 anos os mais propensos. Por sua vez, Estrela et al. (2018) evidenciaram que os estudantes sem bolsa de estudo ou sem vínculo empregatício tiveram maiores pontuações de stress.

Tal igual neste estudo, os resultados de Assis, Silva, Lopes, Silva \& Santini (2013), Vieira e Schermann (2015) e Cestari et al. (2017, demonstraram que estudantes do gênero feminino apresentaram maiores escores em stress. Muito recentemente, Aliante et al. (2019), observaram que ser solteiro, desempregado e mais novo constituíam indicadores de maior vulnerabilidade em stress, pois os sujeitos com essas características obtiveram mais pontuações nas diversas sintomatologias de stress principalmente nas fases de alerta e resistência. Graner e Cerqueira (2019), entendem que as condições socioeconômicas desfavoráveis, acentuadas pela ainda vigente discriminação de gênero em muitas culturas, podem contribuir para o sofrimento psíquico das mulheres.

Em contraponto aos resultados obtidos neste estudo, Assis, Silva, Lopes, Silva \& Santini (2013) indicaram que os sujeitos com idades entre 36 a 42 anos sinalizaram maiores níveis de stress. Também Cestari et al. (2017) notaram que estudantes com maiores chances de apresentar stress tiveram o seguinte perfil: morar com companheiro e frequentar último ano da faculdade. $\mathrm{Na}$ mesma perspectiva, Costa et al. (2018) identificaram alta prevalência de stress entre os estudantes casados.

\section{Considerações finais}

Coube este estudo avaliar os sintomas de stress em estudantes do curso de Psicologia de uma Universidade pública da região sul de Moçambique, verificando pos- 
síveis associações com as variáveis sociodemográficas e acadêmicas. Os resultados permitem depreender que os estudantes pesquisados se sentem stressados, com predomínio de sintomas de natureza psicológica. Também sinalizam que a maior parte dos participantes desenvolve sintomas da fase de resistência. No entanto, é crucial considerar os sintomas psicológicos assinalados na fase de exaustão, uma vez que dos 11 que compõe a escala aplicada, cinco foram mais recorrentes.

Uma vez que os achados deste estudo sinalizam a ocorrência de stress nos estudantes, é necessário no plano institucional, a implantação de um programa de prevenção e intervenção direcionado a toda a comunidade estudantil. Essa medida se torna urgente e importante, pois um dos perigos da ausência de estratégias de prevenção e intervenção é a cronicidade do stress no contexto acadêmico dos estudantes. A este respeito, tal como outros autores (Pereira et al., 2019; Vieira \& Schermann, 2015) propõe-se o incentivo das atividades de prevenção através da promoção de políticas institucionais para que os acadêmicos desenvolvam repertório de manejo e prevenção do stress. Tais ações podem ser através de criação de espaços institucionais para a discussão e a solução dos problemas enfrentados pelos alunos, de modo que a experiência acadêmica seja vivenciada não de forma isolada, mas como um processo que envolve os alunos e também os professores e a instituição como um todo (Bardagi \& Hutz, 2011).

Ao final deste artigo importa mencionar que o desenho da pesquisa não permite generalizar os resultados para todos os estudantes moçambicanos, pois é do tipo transversal e esta se realizou num contexto sociocultural singular, envolvendo uma amostra de um único curso. Assim, sugere-se, para futuros estudos, envolver amostras maiores e diversificadas, objetivando identificar os fatores de stress acadêmico e estratégias de coping. Dessa forma, poder-se-á compreender melhor o fenômeno de stress e propor medidas de prevenção e/ou intervenção que podem contribuir na promoção da saúde mental e física dos estudantes.

\section{Referências}

Abacar, M. (2011). Stress ocupacional e o bem-estar de professores do ensino básico em escolas moçambicanas. (Dissertação do Mestrado). Faculdade de Psicologia e Ciências da Educação, Universidade do Porto, Porto.

Aguiar, S. M., Vieira, A. P. G. F., Vieira, K. M. F., Aguiar, S. M., \& Nóbrega, J. O. (2009). Prevalência de sintomas de estresse nos estudantes de medicina. Jornal Brasileiro de Psiquiatria,58, 34-38. https://doi.org/10.1590/ S0047-20852009000100005

Aliante, G., Abacar, M., Saquina, B. H., \& Aristides, C. L. (2019). Stress em estudantes de graduação em uma universidade pública da região norte de Moçambique. Trabalho En(Cena), 4(2), 465-484. https://doi. org/10.20873/2526-1487V4N2P465
Almeida, H., Brito-Costa, S. Alberty, A., Gomes, A., Lima, P., \& Castro, F. V. (2016). Modelos de stress ocupacional: Sistematização, análise e Descrição. INFAD - Revista de Psicología, 2(1), 435-454.http://dx.doi.org/10.17060/ ijodaep.2016.n1.v2.309

Ariño, D. O., \& Bardagi, M. P. (2018). Relação entre fatores acadêmicos e a Saúde Mental de estudantes universitários. Psicologia em Pesquisa, 12(3), 4452. http://dx.doi.org/10.24879/2018001200300544

Assis, C. L., Silva, A. P. F., Lopes, M. S., Silva, P. C. B., \& Santini, T. O. (2013). Sintomas de estresse em concluintes do curso de psicologia de uma faculdade privada do norte do País. Mudanças - Psicologia da Saúde, 21(1), 23-28. http:/ /dx.doi.org/10.15603/2176-1019/mud.v21n1p23-28

Associação Nacional dos Dirigentes das Instituições Federais de Ensino Superior (ANDIFES) (2019). V Pesquisa Nacional de Perfil Socioeconômico e Cultural dos (as) Graduandos (as) das IFES. Uberlândia.

Bardagi, M. P.,\& Hutz, C. S. (2011). Eventos estressores no contexto acadêmico: Uma breve revisão da literatura brasileira. Interação em Psicologia, 15(1), 111-119. http://dx.doi.org/10.5380/psi.v15i1.17085

Bayram, N., \& Bilgel, N. (2008). The prevalence and sociodemographic correlations of depression, anxiety and stress among a group of university students. Society Psychiatry \& Psychiatry Epidemiology,43, 667-72. https://doi. org/10.1007/s00127-008-0345-x

Bublitz, S., Guido, L. A., Lopes, L. F. D., \& Freitas, E. O. (2016). Associação entre estresse e características sociodemográficas e acadêmicas de estudantes de enfermagem. Texto Contexto em Enfermagem, 25(4), 1-7. http://dx.doi. org/10.1590/0104-07072016002440015

Burrows, M. T. (2004, October). El Estrés y la Salud Mental en el Trabajo: Documento de Trabajo de la Asociación Chilena de Seguridad. Ciencia \& Trabajo, 185-188. http://dx.doi.org/10.1590/0104-07072016002440015

Carleto C. T., Moura, R. C. D, Santos, V. S., \& Pedrosa, L. A. K. (2018). Adaptação à universidade e transtornos mentais comuns em graduandos de enfermagem. Revista Electrónica de Enfermagem, 20(20), 1-11. doi: https:// doi.org/10.5216/ree.v20.43888

Cestari, V. R. F., Barbosa, I. V., Florêncio, R. S., Pessoa, V. L. M. P., \& Moreira, T. M. M. (2017). Stress em estudantes de enfermagem: estudo sobre vulnerabilidades sociodemográficas e acadêmicas. Acta Paulista de Enfermagem, 30(2), 190-6. http://dx.doi.org/10.1590/19820194201700029

Chaves, L. B., Souza, T. F., Silva, M. V. C., Oliveira, C. F., Lipp, M. E. N. \& Pinto, M. L. (2016). Stress em universitários: análise sanguínea e qualidade de vida. Revista Brasileira de Terapias Cognitivas, 12(1), 20-26. http://dx.doi. org/10.5935/1808-5687.20160004

Costa, C. R. B., Maynart, W. H. C., Oliveira, L. B., Albuquerque, M. S. C., \& Correia, D. S. (2018-set./dez.). Stress entre estudantes de graduação em enfermagem: Associação de características sociodemográficas e acadêmicas. Saúde e Pesquisa, 11(3), 475-482. https://doi.org/10.17765/2176-9206.2018v11n3p475-482

Estrela, Y. C. A., Rezende, A. C. C. Guedes, A. F., Pereira, C. O., \& Sousa, M. N. A. (2018). Stress e correlatos com características de saúde e sociodemográficas de estudantes de medicina. Revista CES Médica, 32(3), 215-225. http://dx.doi.org/10.21615/cesmedicina.32.3.3

Faiad, C., Souza, V., Matsunaga, L. H., Rodrigues, C. M. L., \& Rosa, H. R. (2018). Propriedades psicométricas do ISSL no contexto da segurança pública. Estudos Interdisciplinares em Psicologia, 9(3), 54-72. http://dx.doi. org/10.5433/2236-6407.2018v9n3suplp54

Graner, K. M., \& Cerqueira, A. T. B. R. (2019). Revisão integrativa: sofrimento psíquico em estudantes universitários e fatores associados. Ciência \& Saúde Coletiva, 24(4), 1327-1346. https://doi.org/10.1590/141381232018244.09692017

Lima, R. L., Soares, M. E. C., Prado, S. N., \& Albuquerque, G. S. C. (2016). Estresse do estudante de medicina e rendimento acadêmico. Revista Brasileira de Educação Médica, 40(4), 678-684. http://dx.doi.org/10.1590/1981$-52712015 \mathrm{v} 40 \mathrm{n} 4 \mathrm{e} 01532015$

Limongi-França, A. C. \& Rodrigues, A. L. (2009). Stress e trabalbo: uma abordagem psicossomática, (4a . ed., 3. reimp.) São Paulo: Atlas.

Lipp. M. E. N. (2004). Stress emocional: esboço da teoria de "Temas de Vida". In M. E. N. Lipp (Org.). O stress no Brasil: pesquisas avançadas (pp. 17-30). Campinas: Papirus.

Lipp, M. E. N. (2016). O Stress do professor frente ao mau comportamento do aluno. In D. C. Fava (Org.). A Prática da Psicologia na Escola (pp. 351 37). Belo Horizonte: Artesã. 
Lipp, M. E. N., Costa, K. R. S. N., \& Nunes, V. O. (2017). Estresse, qualidade de vida e estressores ocupacionais de policiais: Sintomas mais frequentes. Revista Psicologia: Organizações e Trabalho, 17(1), 46-53. http://dx.doi. org/10.17652/rpot/2017.1.12490

Martins, B. G., Silva, W. R. da., Maroco, J., \& Campos, J. A. D. B. (2019). Escala de Depressão, Ansiedade e Estresse: Propriedades psicométricas e prevalência das afetividades. Jornal Brasileiro de Psiquiatria, 68(1), 32-41. https://doi.org/10.1590/0047-2085000000222

Medeiros, M. R. B., Camargo, J. F., Barbosa, L. A. R., \&Caldeira, A. P. (2018). Saúde mental de ingressantes no curso médico: Uma abordagem segundo o sexo. Revista Brasileira de Educação Médica, 42(3), 214-221. http://dx.doi. org/10.1590/1981-52712015v42n3rb20170008

Moretti, F. A. \& Hübner, M. M. C. (2017). O estresse e a maquina de moer alunos no ensino superior: Vamos repensar a nossa Política Educacional? Revista Psicopedagogia, 34(105), 258-67. Disponível em: http://pepsic.bvsalud. org/scielo.php?script=sci_arttext\&pid=S0103-84862017000300003

Nodari, N. L., Flor, S. R. A., Ribeiro, A. S., \& Carvalho, G. J. R. (2014). Estresse, conceitos, manifestações e avaliação em saúde: revisão de literatura. Revista Saúde e Desenvolvimento Humano, 2(1), 61-74. http://dx.doi. org/10.18316/1543

Nogueira, J. M., \& Gomes, A. R. (2014). Compreender o estresse no esporte: Contribuição das perspectivas transacionais sobre a adaptação humana. In J. M. Montiel, D. Bartholomeu, \& M. S. Andrade (Eds.). Perspectivas em aprendizagem bumana (pp. 57-82). São Paulo: Memnon Edições.

Pereira, R. R., Faciola, R. A., Ramos, M. F. H., Silva, S. S. C., Pontes, F. A. R., \& Ramos, E. M. L. S. (2019). Estresse e características resilientes em alunos com deficiência e TFE na UFPA. Psicologia: Ciência e Profissão, 39, e180093, 1-16. https://doi.org/10.1590/1982-3703003180093

Querido, I. A., Naghettini, A. V., Orsini, M. R. C. A.,Bartholomeu, D.,Montie, J. M. (2016). Fatores associados ao estresse no internato médico. Revista Brasileira de Educação Médica, 40(4), 565-573. http://dx.doi.org/10.1590/1981$-52712015 \mathrm{v} 40 \mathrm{n} 4 \mathrm{e} 00072015$
Salomão, I. F. C. S., Abacar, M., \& Aliante, G. (2018). Satisfação acadêmica em estudantes de Ensino Superior: Um estudo no curso de graduação em ensino básico da Universidade Pedagógica-Delegação de Nampula. Revista Brasileira de Ensino Superior, 4(1), 24-42. http://doi. org/10.18256/2447-3944.2018.v4i1.2239

Selye, H. (1993). History of stress concept. In L. Goldberg \& S. Briznitz (Eds.). Handbook of stress. Theoretical and clinical aspects. New York: The Free Press.

Silva, L. C., \& Salles, T. L. A. (2016). O estresse ocupacional e as formas alternativas de tratamento. ReCaPe - Revista de Carreiras e Pessoas, 6(2), 234 247. https://doi.org/10.20503/recape.v6i2.29361

Siqueira, A. E. O. S., Holzmann, C. S., Amorim, C. A. A., \& Moser, A. M. (2017). Avaliação do nível de stress em acadêmicos dos cursos de ciências sociais e Pedagogia da turma noturna de uma instituição particular. In Anais do IV Seminário Internacional de Representacōes Sociais, Subjetividade, e Educașão, VI Seminário Internacional sobre Profissionalização Docente (ISSN: 2176-1396), (pp.17451-17564). Disponível em: https://educere.bruc.com.br/arquivo/ pdf2017/27120_14022.pdf

Souza, R. G., Santana, E. B., Pedra, R., Dias, D., Henrique, E., \& Dantas, M. (2015). A relevância dos instrumentos de avaliação de ansiedade, estresse e depressão. Ciências Biológicas e de Saúde, 3(1), 37-57. Disponível em: https:// periodicos.set.edu.br/index.php/cadernobiologicas/article/view/2493

Vieira, L. N. \& Schermann, L. B. (2015). Estresse e fatores associados em alunos de psicologia de uma universidade particular do sul do Brasil. Aletheia, 46, 120-130. Recuperado de http://pepsic.bvsalud.org/scielo. php?script $=$ sci_arttext\&pid $=$ S1413-03942015000100010

Submetido em: 13-4-2020

Aceito em: 28-12-2020 